\title{
Gigantiform Cementoma
}

National Cancer Institute

\section{Source}

National Cancer Institute. Gigantiform Cementoma. NCI Thesaurus. Code C8381.

A rare, benign, slow growing, multifocal, and expansile lesion of the jaws. Both familiar and sporadic cases have been reported. It is characterized by a fibroblastic proliferation, cementum, and limited bone formation. 